\title{
Research on Rapid Design Method for Complex Product Based on General Structure Model and Reusable Parts Resource
}

\author{
$\mathrm{Li} \mathrm{He}^{*}$ and Wenlei Sun
}

School of Mechanical Engineering, Xinjiang University, Urumqi, 830047, China

\begin{abstract}
Because of a few characteristics of complex product, such as the variety of its components, and the structural complexity and so on, the realization of the rapid design of complex product requires combination of a variety of theories and methods that support product's rapid design. Therefore, this study assesses complex products and puts forward a new rapid design method based on general structure model and reusable parts resource. First of all, the characteristics and implementation process of this method is summarized. Then, the general structure model of the complex product family is established on the basis of modular thoughts, and the definitions of the four vari-size grained abstract concepts are discussed in this model as well as its semantic relationships. The configuration design of the complex products is based on this general structural model, and with the increase of networked product design information, and more and more manufacturing enterprises have built up their own web-based component repositories to store those detailed design information. On account of those, the rapid design process of the complex product is introduced, with configuration design and reusable parts resource as the core. Lastly, an application example (i.e. the conventional beam pumping unit) is presented to verify the feasibility of which has been discussed above.
\end{abstract}

Keywords: Complex product, General structure model, Product rapid design, Reusable parts resource.

\section{INTRODUCTION}

Product design process must be flexible and agile enough to shorten the development cycle effectively because of diversification in customer's demand and quick product renewal. The Rapid Design (RD), also known as Agile Design (AD) or Rapid Response Design (RRD), which is the extension of advanced manufacturing and CAD/CAM technology [1], has the following characteristics: short product design cycle, high resource reuse rate and fast response speed to customers' requirements. Currently, the main theories or methods supporting the product rapid design are as follows [2-4]: the digital/virtual prototype design, the networked collaborative design, the modular design, and the intelligent design which is based on knowledge engineering and the derived variant design, configuration design and so on. Among them, the configuration design is realized on the basis of the standardized and generalized product structure model [5].

Whichever rapid design methods is used, its core thought is to make the most of abundant knowledge resources accumulated in the enterprise's long-term production activities, so as to manufacture high quality and low cost products efficiently in order to meet the changing market's demand. It has been estimated that $90 \%$ of the industrial design activity is based on variant designs, while during a redesign activity up to $70 \%$ of the information and knowledge is taken from the previous solutions [6]. Consequently, reusing and sharing design resources is a key

*Address correspondence to this author at the School of Mechanical Engineering, Xinjiang University, Yan'an Road, Urumqi, 830047, China; Tel: +86-13659902828; E-mail: heli66@163.com to responding to markets quickly in enterprises. As we know, web-based parts repository is an organization form of reusable component design resources, and support parts' standardization and normalization. Based on the 3D CAD model library and database, it not only integrates the CAD system to realize parametric design of the part online, but also supplies a series of part family to structure the product family, so as to support the rapid design of the product in a design-reuse method.

Nevertheless, the complex product is a kind of product that has various and a huge number of components, has complicated construct, and needs a large quantity of resource for manufacturing and assembling. Because of its complexity, multiple theories and methods, such as the configuration design, variant design, etc., should be combined to realize its rapid design.

Therefore, this study assessed complex products and put forward a new rapid design method based on general structure model and reusable parts resource. It has two steps: the first one which has been discussed in the second section is the modularization of product's structure-that is building up the general structure model of complex product family with modularized method, and based on it to realize rapid configuration design. The other one discussed in the third section is the case-reuse and variant design of componentsthat is invoking the large number of $3 \mathrm{D}$ CAD models resource interior or outside of enterprise directly with the networked parts repository as platform, or utilizing the online parameterized driving function to realize variant designs of the components to get new parts to satisfy different users' demands. 


\section{GENERAL STRUCTURE MODEL OF COMPLEX PRODUCT FAMILY AND CONFIGURATION DESIGN}

The configuration design is one of the many advanced product rapid design methods, while the premise of the product resources management, configuration design and variant design is a reasonable general structure of the complex product family. The modularity of complex product has the following characteristics: firstly, it is composed of a series of complex components, and divided into several general modules according to the different functions in the product. Secondly, the architectural feature and implementation principle of the general module implementing a specific function could be varied and is configurable; thirdly, each product's configuration module (that is also an instantiated simple module) is composed of a series of simple parts. Based on these characteristics, we can build a configurable general structure model of complex product family as shown in Fig. (1).

There are four varying-size grained abstract concepts introduced into this model, those are complex product family, product common module (expressed by $M_{i}$ ), product simple module (expressed by $M_{i j}$ ) and simple component. Their definitions and semantic relationships are as follows:

Definition 1: A complex product family is a master product structure tree, composed of several abstraction levels such as common product module, simple product module and simple component, and is expressed by $P S$.

Definition 2: A product common module, also named the configuration module, is used as an umbrella term for the structural unit which could implement a function and whose structural shape is relatively independent. They are classified by their functions and expressed by $P M$. "Composition relationship" exists between $P M$ and $P S$, expressed as $P M=$ Part_Of $(P S)$, that is $P M_{i} \subseteq P S, i=1,2, \cdots, n$.

Definition 3: A simple product module, also named as the optional module, is a specific structural unit which could be implemented on a particular function. They are classified by their implementation principles and architectural features and they are expressed by $P C$. "Optional relationship" exists between $P C$ and $P M$, expressed as $P C=I S_{-}$Optional $(P M)$, that is $P C_{j} \subseteq P M, j=1,2, \cdots, n$.

Definition 4: A simple component is a minimum structural unit of the product module, expressed by $P P$. "Assembly relationship" exists between $P P$ and $P C$, expressed as $P P=A s s_{-} O f(P C)$, that is $P P_{k} \subseteq P C, k=1,2, \cdots, n$.

As stated, the common structure model describes a series of complex products (namely complex product family) which have a similar structure and function. The

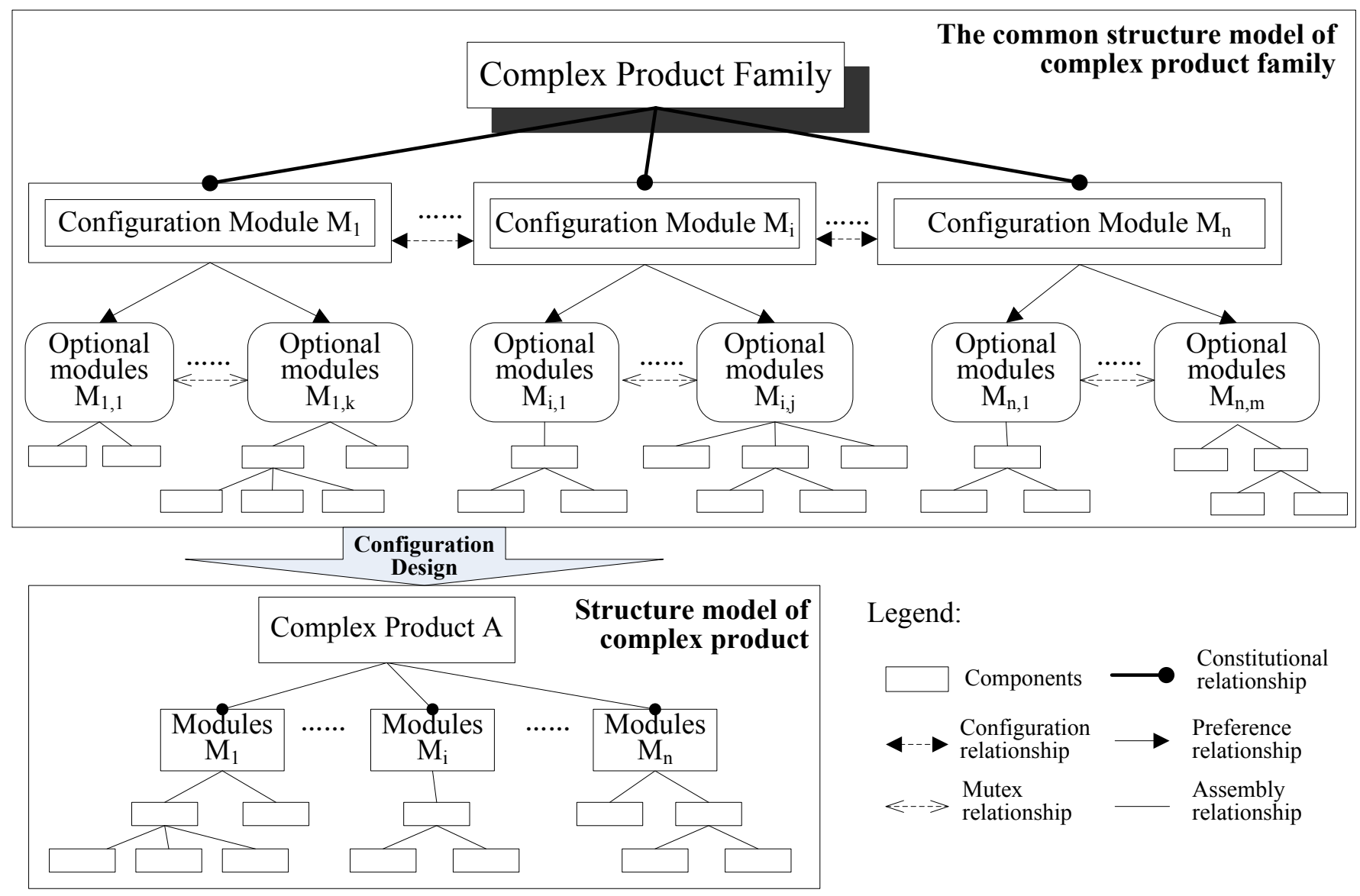

Fig. (1). General structure model of complex product family and configuration design. 
configuration relationship exists between each configuration module, while the match of the optional module is determined by users' different demands for function and performance of the product [7]. Therefore, based on this general model, the product configuration design is a complicated process that configures a product structure's instance with design rules in order to satisfy the customers' requirements. And each specific structural model reflects structural relationships between component instances. When the requirements changes, we can modify the product's structure to satisfy the rapidly changing market demand easily by using the method of product configuration design.

\section{COMPLEX PRODUCT RAPID DESIGN MODE BASED ON REUSABLE PARTS RESOURCE}

A survey shows that, although the customer-oriented product changes quickly, the parts constituting the products are relatively stable. In a new product development process, about $40 \% \sim 50 \%$ of the parts are reused, $30 \% \sim 40 \%$ are modified above the existing ones and only $10 \% \sim 20 \%$ are brand new designs [8]. And so they come under complex product design. Therefore, it is necessary to reuse the existing parts resource possibly to shorten the product development cycle.
With the increase of networked product design information, more and more manufacture enterprises have begun to build up their own web-based component repositories to store those detailed design information [9]. Generally speaking, the web-based component repository is a kind of service platform to share design knowledge and to supply services for reusing and sharing parts resource, where each part takes a unified description form so as to share and exchange parts and products information (3D CAD models of components, e.g.) between the cooperators. Furthermore, it could provide the standard argument list and online parametric driven design services to realize the variant design and configuration design. So making full use of the parts resource in repository is another means of realizing the product's rapid design with the configuration design on the basis of general structure as the core. The application mode of the web-based component repository for complex product rapid design is shown in Fig. (2).

In the web-based component repository, there are mainly two kinds of integration modules that are involved: the first adopts the mediators for distributed part resource library that have referred to PLIB ontology (officially the ISO 13584 Standard series "Parts Library", which has defined a model and an exchange format for digital libraries of technical components [10]) to realize the basic ontological driving automatic integration, and generate an integration

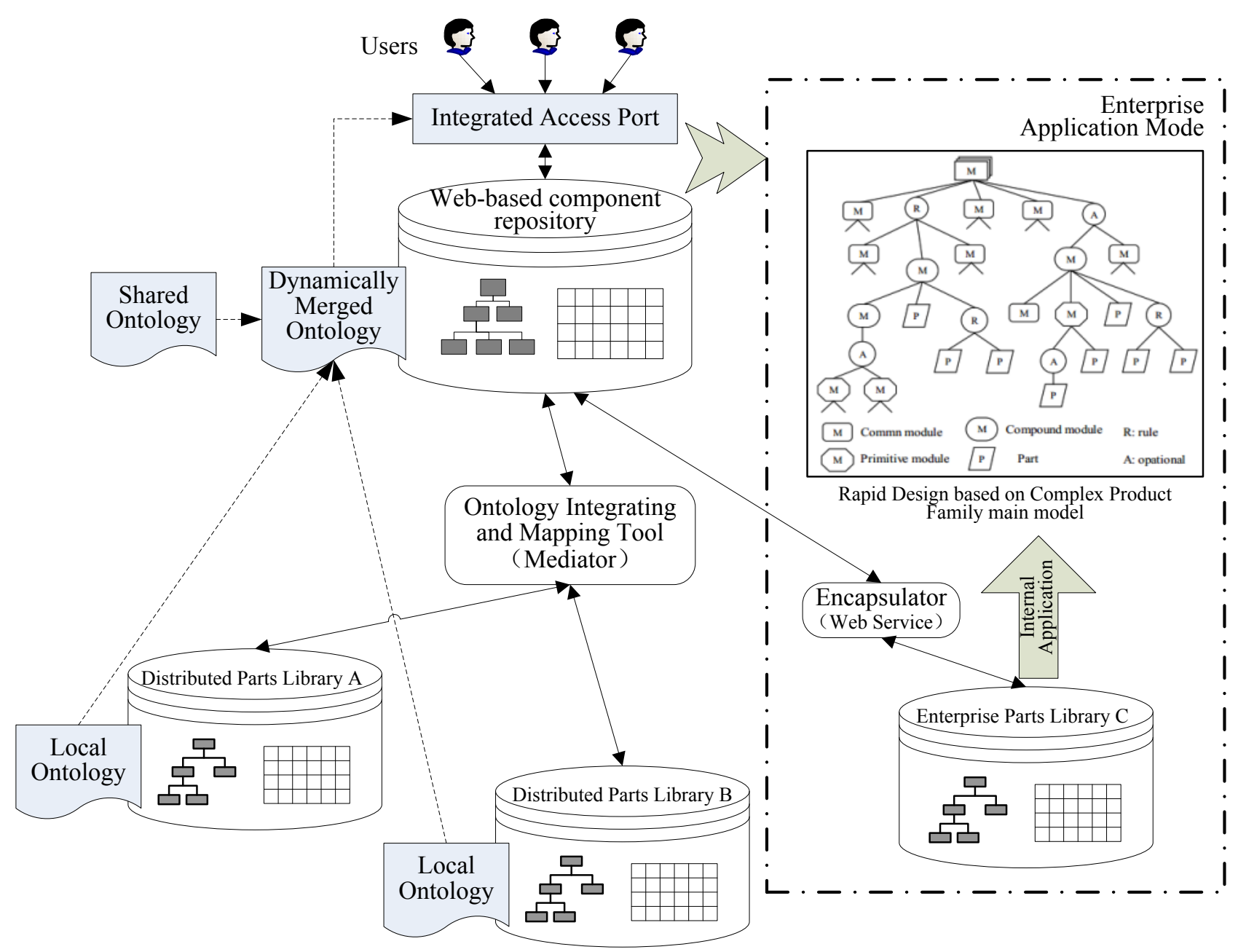

Fig. (2). The application mode of web- for complex product rapid design. 
connector to the distribution part library by dynamic integration; the second is as for those heterogeneous part library resources that have strong independence to utilize companies' established part library resource as much as possible, and can use Web Service encapsulator to seal and package so that the loose coupling virtual integration can be realized.

On the basis of the main structure model of complex product family, enterprise users could directly obtain the required part resource from his local parts library or the webbased component repository through integrated interface, along with the 3D model and design information of parts, to develop a new product.

The main implementation processes (see Fig. 3) are as follows:

Step 1: According to the configuration rules and the general structure model of complex product, determine the structure module of product which could meet the customers' requirements.

Step 2: With the unified retrieval entrance offered by the web-based component repository, get the satisfactory (or high similar) part family and its instance, using a certain matching algorithm.

Step 3: Take the acquired part instance as the unit set, and reconstruct a specific product model according to the certain assembly constraint rules.

In the reusing parts resource process, for those complete matching parts, they could be acquired from the component's repository directly, while for similar parts, they could be adopted after using variant design. When those parts are chosed and inserted into a specific product structure, its relevant information such as supplier, materials and geometrical features etc., will be associated with the specific complex product structure model by reference relationship (Case_of), while the 3D CAD models of the parts could be used directly in the Virtual Prototyping design of complex products. It is an effective measure to shorten the existing component's modeling time and reduce the error rate, and increase the support for the product rapid design.

\section{APPLICATION EXAMPLE}

Based on the method discussed above, we built a general structure model of complex product family for the conventional beam pumping unit (shown in Fig. 4).

As the general model of the pumping unit shows, the Case_of relationship may be built between simple components (seen in definition 4) and the existing parts, that is to build a mapping relation between the common classification level of parts resource and the structure level of complex product, to realize the rapid reuse and variant design of the standard/common components. The Fig. (5) shows an instance of complex product after configuration designing, and a referenced instance of the standard/common parts resource from web-based repository.

In the rapid design process, the standard/common parts resource could be acquired from web-based repository directly through Case_of relationship, and the variant design of virtual prototype could be realized through the method

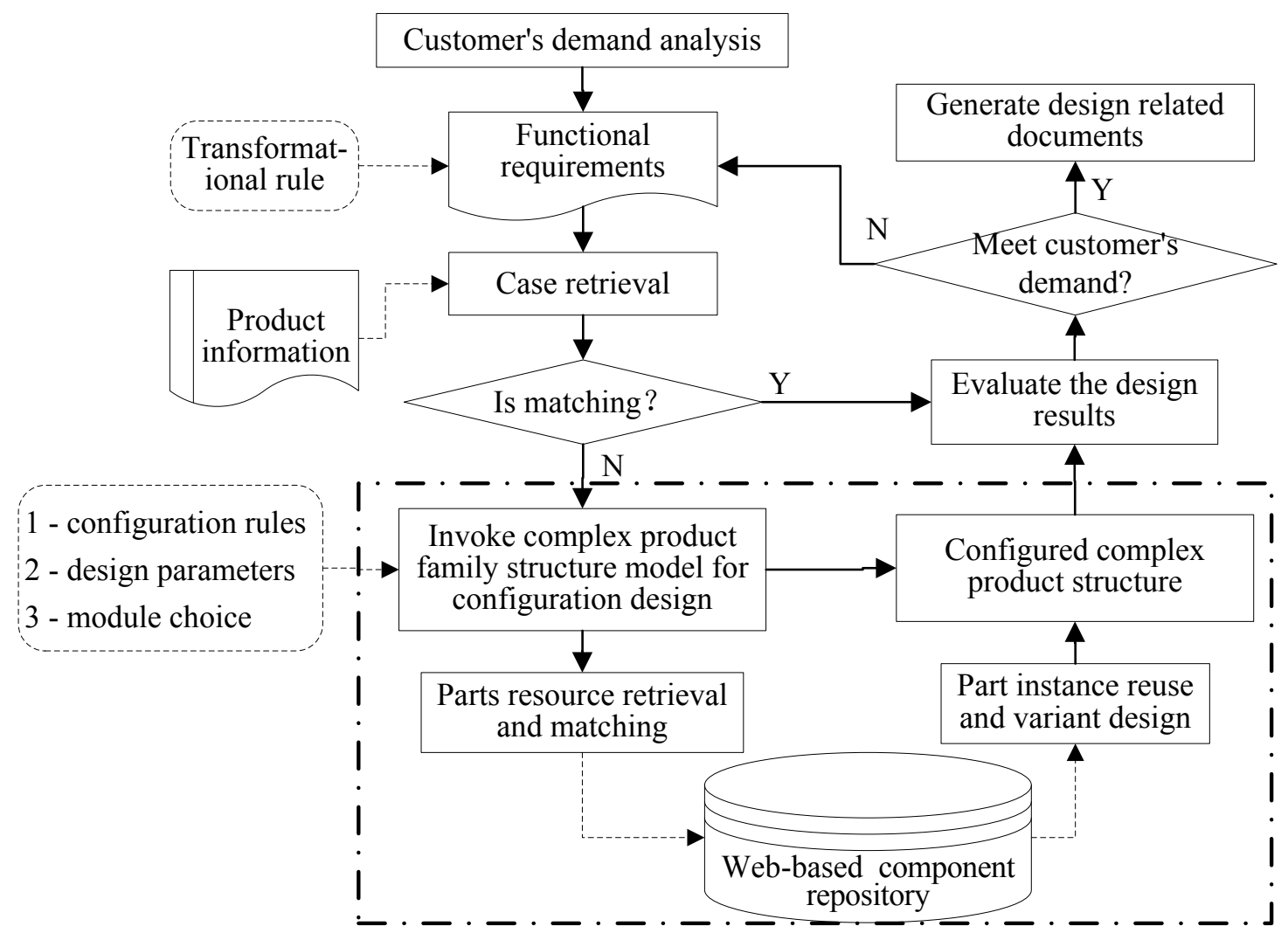

Fig. (3). Rapid design process based on reusable parts resource. 


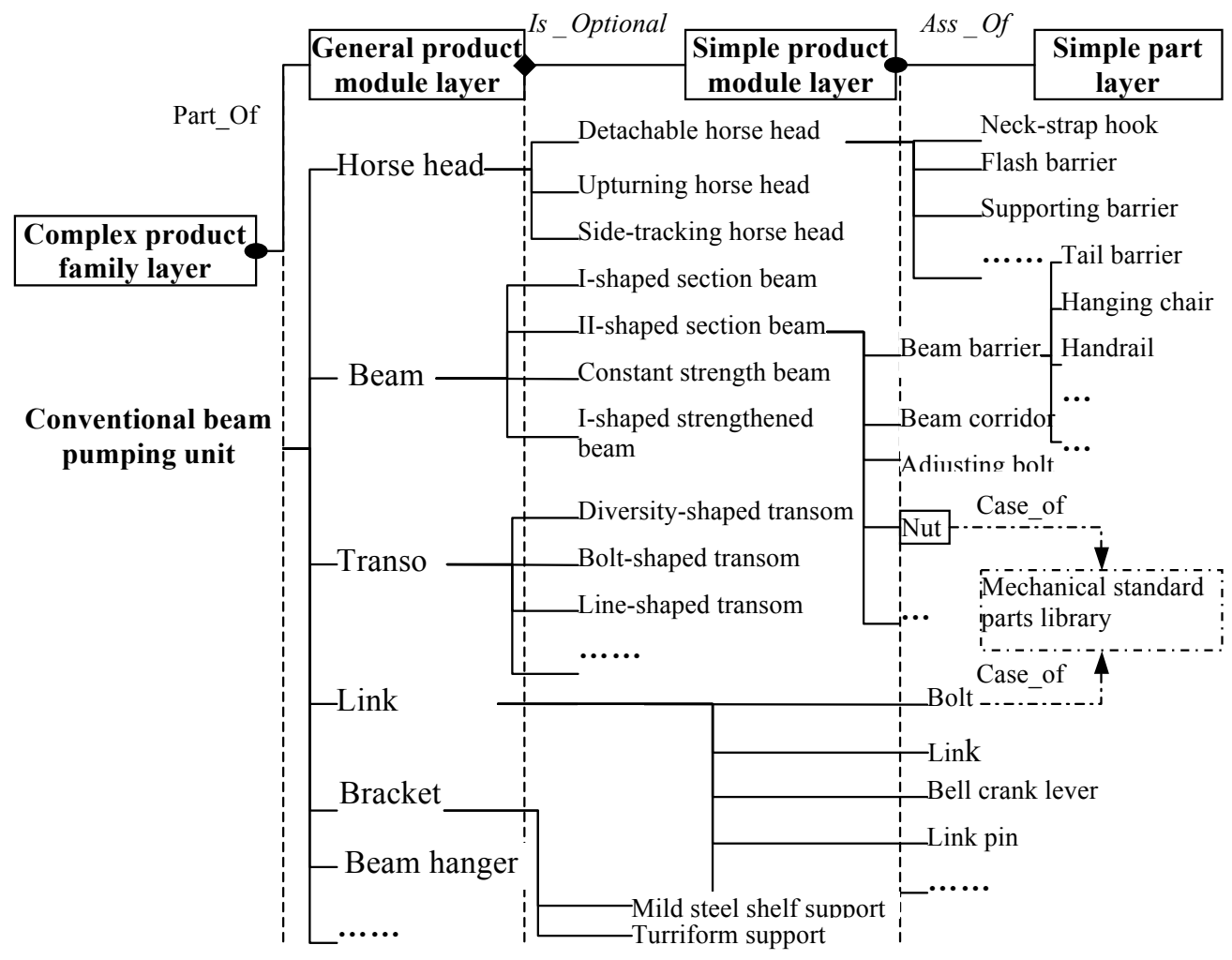

Fig. (4). A general structure model of complex product family for the conventional beam pumping unit.

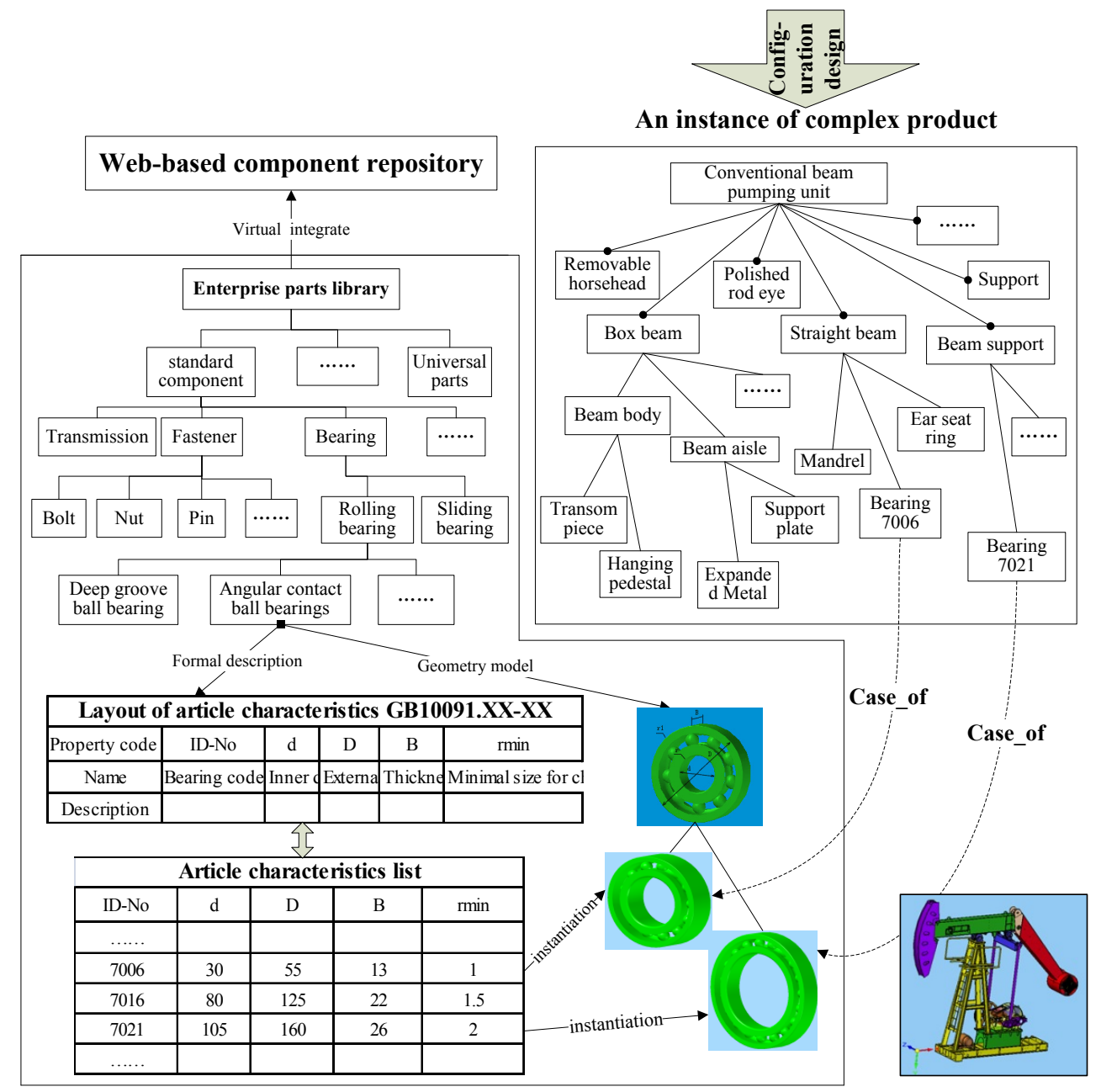

Fig. (5). Application example. 
that obtains new parts within the parameterized drived design. It is also a process to adjust new design demands by improving the existing series of products or design instances, and it can respond to customers' demands for specific functions rapidly and shorten the development cycle for new product.

\section{CONCLUSION}

As discussed above, the complex product rapid design method based on general structural model and part resource reuse consists of two main steps: one is, product structure modularization, which is building complex product family common-use structure model by modularization; the other is standardizing component parts and reusing examples, which is reusing large amount of existing part 3D CAD model resources inside or outside the enterprises' resources libraries directly, or using online parametric driving to realize the variant design of the part resources to satisfy different clients' demands. In product configuration design process, with the help of the functions (parts instances calling, parametric designing, for example) provided by the webbased parts repository, the manufacturing enterprises of complex product could take advantage of the standard/ general components. This method could shorten the modeling time of the parts effectively and reduce the error rate, and then develop diversified and personalized products rapidly. So it can not only shorten product delivery cycle, but also save development costs and improve the product's quality.

\section{CONFLICT OF INTEREST}

The authors confirm that this article content has no conflict of interest.

\section{ACKNOWLEDGEMENTS}

The research work of this paper has acquired support from the following fund programs: Xinjiang University doctor graduate scientific research initial funding (NO. BS130121) and the Xinjiang Uygur Autonomous Region University scientific research planned project (NO. XJEDU2014S008).

\section{REFERENCES}

[1] X. H. Liu and Q. Li, "Development of a rapid design system for aerial work truck sub frame with UG secondary development framework", Procardia Eng., vol. 15, pp. 2961-2965, 2011.

[2] H. Z. Yang, J. F. Chen and N. Ma, "Implementation of knowledgebased engineering methodology in ship structural design," Comput,-Aided Des., vol. 44, pp. 196-202, 2012.

[3] J. Wang, H. L. Zhang and Y. Zhang, "Investigation of key technologies on rapid modular design of bucket elevators", $J$. Mach. Des., vol.29, pp.13-17, 2012.

[4] A. K. W. Lau, R.C. M. Yam and E. Tang, "The impact of product modularity on new product performance: mediation by product innovativeness", J. Prod. Innov. Manag., vol. 28, pp. 270-284, 2011.

[5] L. M. Qi, B. Ren and S. Y. Zhang, "Reconfigurable modeling technology of similar-structure products configuration design", Chin. J. Mech. Eng., vol. 01, pp. 189-196, 2013.

[6] D. Zhang, D. $\mathrm{Hu}$ and Y. C. $\mathrm{Xu}$, "A framework for design knowledge management and reuse for product-service systems in construction machinery industry", Comput. Indust., vol. 63, pp. 328-337, 2012.

[7] Y. D. Wu, G. N. Qi and Q. S. Xie, "Information Modeling for Product Customization Platform Based on Parts Library", In: Proceedings of the $5^{\text {th }}$ Machine Learning and Cybernetics Conference, 2006, pp. 13-16.

[8] J. Ma, G. N. Qi and X. J. Gu, "Parts resource reusable modeling and matching for rapid response design," J. Zhejiang Univ. (Eng. Sci.), vol. 42, pp. 1428-1433, 2008.

[9] B. Jin, H. F. Teng and Y. S. Wang, "Product design reuse with parts libraries and an engineering semantic web for small and medium sized manufacturing enterprises," Int. J. Adv. Manuf. Tech., vol. 38, pp. 1075-1084, 2008.

[10] G. Pierra, E. Sardet, J. C. Potier G. Battier and J. C. Derouet, "Exchange of Component Data: The PLIB (ISO 13584) Model, Standard and Tools", In: Proceedings of the CALS Europe 98 Conference, 1998, pp. 160-176. 